\title{
Pre-intervention characteristics in weight loss participants scoring positive and negative for food addiction
}

\author{
Trina Aguirre ${ }^{1 *}$, Rebecca (Becci) Bowman ${ }^{2}$, Rebecca Kreman $^{2}$, Jeffrey Holloway², Jason LaTowsky ${ }^{2}$, Martha Stricker², Leeza Struwe ${ }^{3}$, Erica \\ Schulte $^{4}$, Ann Koehler ${ }^{1}$, Kayla Pierce ${ }^{1}$ and Molly Bloodgood ${ }^{1}$ \\ ${ }^{1}$ University of Nebraska Medical Center, College of Nursing - West Nebraska Division, 1601 East 27th St., Scottsbluff, NE, USA 69361-1815, USA \\ ${ }^{2}$ Regional West Physicians Clinic, Medical Plaza North, Two West 42nd Street, Scottsbluff, NE 69361, USA \\ ${ }^{3}$ University of Nebraska Medical Center, College of Nursing -Niedfelt Nursing Research Center, PO Box 880220, Lincoln, NE 68588-0220, USA \\ ${ }^{4}$ University of Michigan, Department of Psychology, 2268 East Hall, 530 Church Street, Ann Arbor, MI 48109, USA
}

\begin{abstract}
Obesity is a major health issue in the United States. It has been suggested that addictive-like tendencies toward foods, especially highly processed foods, contributes to this epidemic. If so, interventions used to treat substance-use disorders may be effective for treating overweight/obese patients with food addiction (FA; based on the Yale Food Addiction Scale, version 2.0). This pilot study evaluated four interventions, selected because of their effectiveness in the treatment of substance-use disorders [motivational interviewing (MI), pharmacotherapy (P; naltrexone-bupropion), MI with pharmacotherapy (MI+P), information control (IC; diet and physical activity instruction)], in overweight/obese individuals with and without FA (FA+ and FA-, respectively). Here we report the baseline (pre-intervention) characteristics of FA+ and FA- participants based on their intake documents. FA was fairly common in this population (37.1\% of those screened). Most participants experienced depression $(81.9 \%, \mathrm{FA}+94.3 \%, \mathrm{FA}-73.0 \%)$ and anxiety $(60.2 \%, \mathrm{FA}+74.3 \%, \mathrm{FA}-50 \%)$ with greater prevalence $(\mathrm{p}<.01)$ and severity in those who were FA+. Many participants screened positive for binge eating $(42.2 \%, \mathrm{FA}+65.7 \%, \mathrm{FA}-25.0 \%)$ and to a lesser extent PTSD $(18.1 \%, \mathrm{FA}+37.1 \%, \mathrm{FA}-4.2 \%)$, with greater prevalence among those who were FA+ (p<.01). Drug abuse (20.5\%) and mood disorder (8.4\%) were relatively uncommon and prevalence did not differ between FA phenotypes (p>.05). The FA construct identified a distinctive subset of overweight/obese individuals. Differences in baseline characteristics suggest that FA+ and FA- individuals may differ in their response to interventions and the types of support they need to achieve their weight/body fat loss goals.
\end{abstract}

\begin{abstract}
Abbreviations: Food addiction (FA), food addiction positive $(\mathrm{FA}+)$, food addiction negative (FA-), motivational interviewing (MI), pharmacotherapy $(\mathrm{P})$, MI with pharmacotherapy $(\mathrm{MI}+\mathrm{P})$, information control (IC), Yale Food Addiction Scale (YFAS), Healthy Behaviors Clinic (HBC), Regional West Physicians Clinic (RWPC), Weight and Lifestyle Inventory (WALI), Patient Health Questionnaire-9 (PHQ-9), Generalized Anxiety Disorder-7 (GAD-7), Drug Abuse Screening Test10 (DAST-10), Mood Disorder Questionnaire (MDQ), Posttraumatic Stress Disorder (PTSD) Checklist for DSM-5 (PCL-5), Doctor of Nursing Practice (DNP)
\end{abstract}

\section{Introduction}

Obesity is a major health issue in the United States and is associated with increased risk of comorbidities (hypertension, diabetes, cardiovascular disease, lipid disorders, depression, anxiety, etc.) and increased medical expenditures (approximately $42 \%$ higher for obese than for normal weight individuals) [1]. Numerous approaches have been tried to address obesity with limited long-term success [2-8]. Recently, it has been suggested that addictive-like tendencies toward foods, especially highly processed foods that are high in fat and sugar, contribute to this epidemic [4-7]. The Yale Food Addiction Scale (YFAS) is a relatively new, validated instrument that can help researchers and practitioners assess clients for food addiction (FA) and has been used often in populations with obesity [8,9]. In recent studies, $20-25 \%$ of overweight (BMI 25-29)/obese (BMI $\geq 30$ ) persons tested positive for FA $[7,10]$. However, no known obesity interventions specifically target individuals who are positive for FA. If an addictive-like process contributes to obesity for some individuals, then interventions used to treat substance-use disorders may be effective for the treatment of FA [11].

Therefore, we initiated a pilot study to evaluate four interventions, selected because of their effectiveness in the treatment of substanceuse disorders [individual motivational interviewing alone (MI), pharmacotherapy alone (P; naltrexone-bupropion), MI with pharmacotherapy $(\mathrm{MI}+\mathrm{P})$, and an information control (IC; diet and physical activity instruction)], in overweight/obese individuals with and without FA with the goal of developing effective interventions for each group. This study is unique in purposefully recruiting FA positive (FA+) and FA negative (FA-) participants to evaluate how they may differ in their response to obesity interventions and in evaluating whether substance addiction treatments can be applied successfully to FA. Here we compare the baseline characteristics (pre-intervention) of FA+ and FA- participants based on their intake documents. Such comparisons expand our knowledge of the similarities and differences between overweight/obese individuals with and without addictive-like tendencies towards food, providing insights that may help improve

Correspondence to: Trina Aguirre, University of Nebraska Medical Center, College of Nursing - West Nebraska Division, 1601 East 27th St., Scottsbluff, NE 69361-1815, USA, E-mail: taguirre@unmc.edu

Key words: obesity, food addiction, motivational interviewing, naltrexone, bupropion, drug therapy

Received: July 12, 2018; Accepted: July 24, 2018; Published: July 30, 2018 
obesity interventions and support for both types of individuals. Details of the interventions and their impacts (biometric and dietary) will be reported separately after the conclusion of the study.

\section{Methods}

\section{Participant recruitment}

Participants were recruited from overweight/obese patients referred to the Healthy Behaviors Clinic (HBC) by doctors at the Regional West Physicians Clinic (RWPC) in Scottsbluff, Nebraska, USA and through snowballing. Potential participants completed a set of screening questionnaires as part of the standard $\mathrm{HBC}$ admission process. These included the Weight and Lifestyle Inventory (WALI; 17 sections) [12], Yale Food Addiction Scale (YFAS 2.0; 35 items) [13], Patient Health Questionnaire-9 (PHQ-9; 9 items) [14], Generalized Anxiety Disorder-7 (GAD-7; 7 items) [15], Drug Abuse Screening Test10 (DAST-10; 10 items) [16], Mood Disorder Questionnaire (MDQ; 3 sections) [17], Posttraumatic Stress Disorder (PTSD) Checklist for DSM-5 (PCL-5; 20 items) [18], and a medical history. These instruments were reviewed by a Doctor of Nursing Practice (DNP) who is board certified in Adult Health and Psychiatry to pre-screen potential participants for eligibility.

\section{Eligibility}

Eligible individuals were overweight/obese adults age 19-65 years of either sex and any race/ethnicity who could understand/read English. Because treatments were randomly assigned, they also had to meet criteria specific to the pharmacotherapy interventions $(\mathrm{P}, \mathrm{MI}+\mathrm{P})$ (e.g. restrictions on medications, medical conditions, pregnancy/lactation). $\mathrm{HBC}$ nurse researchers informed those who were eligible about the study and consented those choosing to participate.

\section{Assessment of food addiction and treatment assignment}

The YFAS 2.0 [13], which adapts the eleven DSM-5 diagnostic indicators of substance-use disorders to the consumption of highly processed foods, was used to assess participants' obesity phenotype (FA+ or FA-). Participants with $\geq 2$ symptoms plus impairment/distress were considered FA+. Those with 0 - 1 symptoms and/or no impairment/ distress were considered FA-. Participants within each phenotype were randomly assigned to one of the treatment groups (IC, MI, MI+P, P).

\section{Measures and data analysis}

To compare the characteristics of individuals with and without FA prior to delivery of interventions, we scored the screening instruments in participants' baseline intake documents; the WALI, Section J: Eating Patterns I (binge eating), YFAS 2.0 (FA), PHQ-9 (depression), GAD-7 (anxiety), DAST-10 (drug abuse), MDQ (mood disorder), and PCL5 (PTSD). Descriptive statistics (M, SD) were used to characterize variables. Differences among variables between participants with and without FA were evaluated with Independent t-tests, Mann-Whitney U tests, or Fisher's Exact tests $(\alpha=.05)$. All data analyses were performed using $\mathrm{IBM}^{\circledR}$ SPSS $^{\circledR}$ Statistics (Version 25) software.

\section{Results and discussion}

\section{Recruitment effort/demographics}

We screened 105 individuals. Eighty-three were enrolled in the study, 14 have withdrawn, 8 were ineligible because of their age (2), medications (2), or medical conditions (4). We planned to recruit equal numbers of FA+ and FA- participants, therefore, as the end of the recruitment period neared we were only able to accept individuals who were FA+. Thus, 14 individuals who were FA- were not enrolled. Up to this point attrition has been approximately $10 \%$, however, the study is still in progress, so this value may change.

Of the 83 participants, 74 were women $(89.2 \%)$ and 9 were men (10.8\%). Most were Caucasian $(\mathrm{n}=59,71.1 \%), 23$ were Hispanic (27.7\%), and 1 was black (1.2\%). Average age was $42.7(\mathrm{SD}=12.6)$ and was similar among those who were $\mathrm{FA}+(\mathrm{M}=43.1, \mathrm{SD}=12.7)$ and FA- $(\mathrm{M}=42.3, \mathrm{SD}=12.6)(\mathrm{p}>.05)$. Age, ethnicity, FA characteristics (presence, clinical significance, total symptoms, level of severity) and prevalence of binge eating, depression, PTSD, and mood disorder did not differ by gender $(P>.05)$. Data were insufficient to assess gender differences in drug abuse and anxiety. Genders were pooled for the remaining analyses.

\section{Prevalence and characteristics}

Of the 105 overweight/obese individuals screened, $62.9 \%$ were FAand $37.1 \%$ were FA+. This is greater than the prevalence of FA among overweight/obese individuals in a recent meta-analysis $(\mathrm{M}=24.9 \%$, range $=7.7-56.8 \%$ ) [7]. and is likely greater than the prevalence of FA in the general population as FA prevalence tends to be less among healthy weight individuals $(11.1 \%$, range $=1.6-24.0 \%)$ [7]

Following current withdrawals, our study population is $54.9 \%$ FA$(\mathrm{n}=39)$ and $45.1 \% \mathrm{FA}+(\mathrm{n}=32)$, slightly different than our target of $50 \%(\mathrm{n}=40)$ each, however, study timelines limited us to a 7 -month recruitment period. Average symptom counts were greater for $\mathrm{FA}+(\mathrm{M}$ $=8.0, \mathrm{SD}=2.9)$ than for FA- participants $(\mathrm{M}=1.7, \mathrm{SD}=1.8)(\mathrm{p}<.01)$. Most individuals who were FA+ showed severe levels of FA $(80 \%)$ with the remainder showing moderate $(8.6 \%)$ or mild (11.4\%) levels.

\section{Comparing variables by FA phenotype}

Most participants experienced some level of depression (81.9\%). Depression tended to be more prevalent (FA+ 94.3\%, FA- 73.0\%, $\mathrm{p}<.01$ ) and severe (none: FA+ 5.7\%, FA- 27.1\%; mild: FA+ $8.6 \%$, FA- $41.7 \%$; moderate: FA+ $31.4 \%$, FA- $25.0 \%$; moderately severe: FA+ $31.4 \%$, FA- $6.3 \%$; severe: FA+ $22.9 \%$, FA- $0.0 \%$ ) among those who were FA+. Burmeister et al. [19], and Davis et al. [20], reported similar findings. The high incidence of depression among study participants is consistent with Luppino et al.'s [21], findings of a bidirectional association between obesity and depression (i.e. obesity increases risk of developing depression, depression increases risk of developing obesity). That depression was common in these overweight/obese participants suggests that they may benefit from the individualized support of the MI intervention which addresses ambivalence, overcoming barriers, and setting achievable personal goals.

\section{Developmental regression and epileptiform EEG abnormalities}

We found no association between developmental regression and initial epileptiform EEG abnormalities $(\mathrm{p}=0.50)$. Regression is a salient feature of ASD thought to be a risk factor for the development of epilepsy with conflicting evidence [5,10,14-17]. A retrospective review of 889 children with primary ASD failed to show an increased occurrence in sleep epileptiform EEG abnormalities in children with history of regression as compared to those without regression [5]. However, a 2017 meta-analysis concluded that there might be a weak relationship between history of regression and epileptiform EEG abnormalities [17]. It is possible that our cohort was not sufficiently powered to reveal an association between regression and epileptiform 
EEG abnormalities in children with ASD, however, inconsistencies and lack of clear consensus indicate a need for further research in this area.

The pharmacotherapy interventions $(\mathrm{P}, \mathrm{MI}+\mathrm{P})$ may also support patients with depression as one component (bupropion) is widely used to treat depression.

Similar to depression, most participants experienced some level of anxiety (60.2\%) and it was more common among those who were FA+ (74.3\%) than among those who were FA- $(50 \%)(\mathrm{p}<.01)$. Severity also tended to be greater among FA+ individuals (none $=25.7 \%$, mild $=$ $31.4 \%$, moderate $=25.7 \%$, severe $=17.1 \%)$ than among those who were FA- $($ none $=50.0 \%$, mild $=33.3 \%$, moderate $=12.5 \%$, severe $=4.2 \%)$.

Just under half of all participants (42.2\%) screened positive for binge eating with 2 participants (1 FA+, 1 FA-) not completing the questions. Prevalence of binge eating was greater among FA+ (65.7\%) than among FA- individuals $(25.0 \%)(\mathrm{p}<.01)$. Although there was overlap in the presence of FA and binge eating, that a substantial number of those with FA did not meet the criteria for binge eating and vice versa, suggests that they are distinct attributes. Chao et al. [22] and Ivezaj et al. [23] reported similar findings.

Though PTSD was relatively uncommon among all study participants (18.1\%), over a third of those who were FA+ screened positive for PTSD (37.1\%), a prevalence far greater than among participants who were FA- $(4.2 \%)(\mathrm{p}<.01)$. Brewerton [11], recently reviewed the relationship between FA, PTSD, and other disorders and concluded that FA could be useful as a proxy for trauma history and PTSD.

Other conditions were less frequently observed. Prevalence and severity of drug abuse were generally low (none $=79.5 \%$, low $=16.9 \%$, substantial $=2.4 \%$, one FA- participant did not complete the questions) and prevalence did not differ by FA phenotype ( $\mathrm{p}>.05)$. Mood disorder was also uncommon (negative $=91.6 \%$, positive $=8.4 \%$ ) and did not differ by FA phenotype ( $\mathrm{p}>.05)$.

\section{Conclusion}

Other conditions were less frequently observed. Prevalence and severity of drug abuse were generally low (none $=79.5 \%$, low $=16.9 \%$, substantial $=2.4 \%$, one FA- participant did not complete the questions) and prevalence did not differ by FA phenotype $(\mathrm{p}>.05)$. Mood disorder was also uncommon (negative $=91.6 \%$, positive $=8.4 \%$ ) and did not differ by FA phenotype ( $\mathrm{p}>.05)$.

\section{Acknowledgements}

We thank the RWPC for clinic space and Don Graham, our consulting pharmacist.

\section{Funding}

This work was supported by a Research \& Engagement Competitive Award by the Rural Futures Institute at the University of Nebraska.

\section{References}

1. Finkelstein EA, Trogdon JG, Cohen JW, Dietz W (2009) Annual medical spending attributable to obesity: payer-and service-specific estimates. Health Aff 28: w822-31. [Crossref]

2. Dombrowski S, Knittle K, Avenell A, Araujo SV, Sniehotta F (2014) Long term maintenance of weight loss with non-surgical interventions in obese adults: systematic review and meta-analyses of randomized controlled trials. BMJ 348: g2646.
3. Johns D, Hartmann BJ, Jebb S, Aveyard P (2014) Diet or exercise interventions vs combined behavioral weight management programs: A systematic review and metaanalysis of direct comparisons. J Acad Nutr Diet 114: 1557-1568. [Crossref]

4. Schulte EM, Avena NM, Gearhardt AN (2015) Which foods may be addictive? The roles of processing, fat content, and glycemic load. PLoS One 10: e0117959. [Crossref]

5. Flint AJ, Gearhardt AN, Corbin WR, Brownell KD, Field AE, et al. (2014) Foodaddiction scale measurement in 2 cohorts of middle-aged and older women. Am J Clin Nutr 99: 578-86.

6. Iozzo P, Guiducci L, Guzzardi MA, Pagotto U (2012) Brain PET imaging in obesity and food addiction: current evidence and hypothesis. Obes Facts 5:155-64.

7. Pursey KM, Stanwell P, Gearhardt AN, Collins CE, Burrows TL (2014) The prevalence of food addiction as assessed by the Yale Food Addiction Scale: a systematic review. Nutrients. 6: 4552-4590.

8. Gearhardt AN, Corbin WR, Brownell KD (2009) Preliminary validation of the Yale food addiction scale. Appetite. 52: 430-436.

9. Gearhardt AN, Roberto CA, Seamans MJ (2013) Preliminary validation of the Yale Food Addiction Scale for children. Eat Behav 14: 508-512. [Crossref]

10. Imperatori C, Innamorati M, Contardi A, Continisio M, Tamburello S, et al. (2014) The association among food addiction, binge eating severity and psychopathology in obese and overweight patients attending low-energy-diet therapy. Compr Psychiatry 55: $1358-1362$.

11. Brewerton TD (2017) Food addiction as a proxy for eating disorder and obesity severity, trauma history, PTSD symptoms, and comorbidity Eat Weight Disord-St 22: 241-247.

12. Wadden TA, Foster GD (2006) Weight and lifestyle inventory (WALI). Obesity 14: S3.

13. Gearhardt AN, Corbin WR, Brownell KD (2016) Development of the Yale Food Addiction Scale Version 2.0. Psychol Addict Behav 30: 113-121.

14. Kroenke K, Spitzer RL (2002) The PHQ-9: a new depression diagnostic and severity measure. Psychiatr Ann 32: 509-15.

15. Spitzer RL, Kroenke K, Williams JB, Lowe B, (2006) A brief measure for assessing generalized anxiety disorder: the GAD-7. Arch Intern Med 166: 1092-1097. [Crossref]

16. Skinner HA (1982) The Drug Abuse Screening Test. Addict Behav 7: 363-371. [Crossref]

17. Hirschfeld, RMA, Williams JBW, Spitzer RL, Calabrese JR, Flynn L, et al. (2000) Development and Validation of a Screening Instrument for Bipolar Spectrum Disorder: The Mood Disorder Questionnaire. Am J Psychiatr 157: 1873-1875. [Crossref]

18. https://www.beaconhealthstrategies.com/pcp toolkit/content/PTSD/Screening\%20 Tools/PCL-

19. Burmeister JM, Hinman N, Koball A, Hoffmann DA, Carels RA (2013) Food addiction in adults seeking weight loss treatment. Implications for psychosocial health and weight loss. Appetite 60: 103-110.

20. Davis C, Curtis C, Levitan RD, Carter JC, Kaplan AS, et al. (2011) Evidence that 'food addiction' is a valid phenotype of obesity. Appetite 57: 711-717.

21. Luppino FS, de Wit LM, Bouvy PF, Stijnen T, Cuijpers P, et al. (2010) Overweight, obesity, and depression: a systematic review and meta-analysis of longitudinal studies. Arch Gen Psychiatry 67: 220-229. [Crossref]

22. Chao AM, Shaw JA, Pearl RL, Alamuddin N, Hopkins CM, et al. (2017) Prevalence and psychosocial correlates of food addiction in persons with obesity seeking weight reduction. Compr psychiatry 73: 97-104.

23. Ivezaj V, White MA, Grilo CM (2016) Examining binge-eating disorder and food addiction in adults with overweight and obesity. Obesity 24: 2064-2069.

Copyright: (C2018 Aguirre T. This is an open-access article distributed under the terms of the Creative Commons Attribution License, which permits unrestricted use, distribution, and reproduction in any medium, provided the original author and source are credited. 